\title{
Solderjet Bumping technique used to manufacture a compact and robust green solid-state laser
}

\author{
P. Ribes ${ }^{* 1,2}$, T. Burkhardt ${ }^{1}$, M. Hornaff ${ }^{1}$, S. Kousar $^{2}$, D. Burkhardt ${ }^{1,8}$, E. Beckert ${ }^{1}$, M.Gilaberte $^{3}$, \\ D. Guilhot ${ }^{3,7}$, D.Montes ${ }^{3}$, M. Galan ${ }^{3}$, S. Ferrando ${ }^{3,7}$, M. Laudisio ${ }^{4}$, T. Belenguer ${ }^{5}$, S. Ibarmia ${ }^{5}$, P. \\ Gallego $^{5}$, J.A. Rodríguez ${ }^{6}$, R. Eberhardt ${ }^{1}$, A. Tünnermann ${ }^{1,2}$ \\ ${ }^{1}$ Fraunhofer Institute for Applied Optics and Precision Engineering IOF, Jena, Germany \\ ${ }^{2}$ Friedrich-Schiller-University Jena, Institute for Applied Physics, Abbe Center of Photonics, Jena, \\ Germany \\ ${ }^{3}$ Monocrom S.L., Dept. R\&D, Vilanova i la Geltrú, Spain \\ ${ }^{4}$ LIDAX, Madrid, Spain \\ ${ }^{5}$ National Institute of Aerospace Technology INTA, Torrejón de Ardoz, Spain \\ ${ }^{6}$ Engineering Systems for the Defense of Spain ISDEFE, Madrid, Spain \\ ${ }^{7}$ Authors are now with the Institute of Photonic Science ICFO, Barcelona, Spain \\ ${ }^{8}$ The Author is now with Numerik Jena GmbH, Jena, Germany
}

\begin{abstract}
Solder-joining using metallic solder alloys is an alternative to adhesive bonding. Laser-based soldering processes are especially well suited for the joining of optical components made of fragile and brittle materials such as glasses, ceramics and optical crystals due to a localized and minimized input of thermal energy. The Solderjet Bumping technique is used to assemble a miniaturized laser resonator in order to obtain higher robustness, wider thermal conductivity performance, higher vacuum and radiation compatibility, and better heat and long term stability compared with identical glued devices. The resulting assembled compact and robust green diode-pumped solid-state laser is part of the future Raman Laser Spectrometer designed for the Exomars European Space Agency (ESA) space mission 2018.
\end{abstract}

Keywords: Solderjet Bumping, laser based soldering, adhesive-free, micro-packaging, Raman Laser Spectrometer (RLS), micro-assembly, diode-pumped solid-state laser resonator, ESA Exomars

\section{INTRODUCTION}

The use of compact and miniaturized lasers capable to operate under extreme conditions has become one of the main goals for the laser manufacturers that develop devices for industry, space, medicine and military applications [1]. Such devices use to demand high output power with a high thermal, wavelength and beam stability by minimizing the size and weight via replacing optical clamping methods with different sort of adhesives. Nevertheless organic adhesives used in extreme applications could suffer slow and constant deterioration, resulting in optical components displacements, laser spectrum degeneration, efficiency losses or even failure.

Solderjet bumping technology is used to manufacture a compact and robust green Diode-Pumped Solid-State Laser (DPSSL) to be used for the next Raman Spectroscopy ESA Exomars mission 2018. The assembled laser is able to overcome all the adhesive induced issues and is adequate to achieve all the stringent specifications need for space applications and Raman laser spectrometry applications, as described by Rull et al. [1].

This laser-based soldering technique offers a local and minimized input of thermal energy and thus allows the components joining with very high position accuracy even for fragile and sensitive optical components such as the

Integrated Photonics: Materials, Devices, and Applications III, edited by Jean-Marc Fédéli,

Proc. of SPIE Vol. 9520, 952009 - (c) 2015 SPIE · CCC code: 0277-786X/15/\$18

doi: $10.1117 / 12.2178373$ 
second harmonic generation crystals. Components need a wettable surface metallization which is provided by physical vapor deposition.

\section{SOLDERJET BUMPING}

Solder-joining using metallic solder alloys is an alternative to adhesive bonding. Laser-based soldering processes are especially well suited for the joining of optical components made of fragile and brittle materials such as glasses, ceramics and optical crystals, due to a localized and minimized input of thermal energy. Different techniques of heating solder alloys by laser irradiation are proposed using either thin film solder layers [3], Pick\&Align resistance soldering technique [4] and [5] or the jetting of laser-molten solder droplets [6]. This so called Solderjet Bumping is a technique adapted from flip chip processing of semiconductor devices also allowing for the flux-free and contact-free processing of optical components and 3D-packaging. It uses spherical solder preforms of various soft solder alloys (e. g. tin-based lead-free solders, low melting indium alloys or high melting eutectic gold-tin, gold-silicon or gold-germanium solders) in a diameter range of $60 \mu \mathrm{m}$ to $760 \mu \mathrm{m}$. The solder spheres are transferred from a reservoir to a placement capillary with a conical tip and an inner diameter that is slightly smaller than the diameter of the spheres. After positioning the capillary next to the joining geometry using an articulating robot or a gantry system, the solder alloy is molten by an infrared laser pulse and jetted out of the capillary by the applied nitrogen pressure. The jetting of liquid solder volumes provides a very good thermal contact of the alloy with the components and allows for the joining within complex 3D-integrated geometries. The bond head of the Solderjet Bumper integrates solder volume feeding, reflow and application of liquid solder droplets in a compact device and allows for highly automated and flexible use (Figure 1).

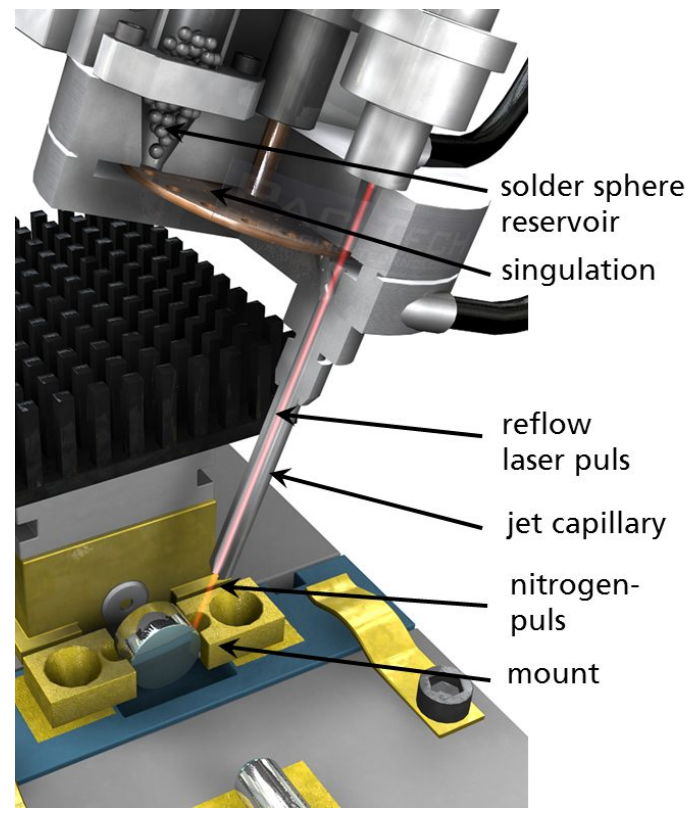

Figure 1: Schematic drawing of the Solderjet bond head able to solder droplets with 6 degrees of freedom (DOF).

The formation of a metallic solder joint using components made of non-metallic materials with Solderjet Bumping requires a wettable metallization layer applied to the components. Such surfaces can be provided by thin film (e. g. physical vapor deposition) or thick film (e. g. screen printing of metal pastes) processes. Sputtered three layer systems using titanium adhesion layer, a platinum diffusion barrier and a noble gold finish preventing oxidization and acting as a wetting surface, provide superb conditions for wetting of liquid solder droplets.

Typical photonic applications of Solderjet Bumping are described in the literature [6]. Sub-micron accuracy in placement of components, direct fiber coupling by soldering of polarization maintaining fibers and the hermetic sealing of an 
endoscopic tip is demonstrated. Further examples show the mounting of sensitive micro-optical components such as gradient-index lenses and fibers. The assembly of a multi-beam deflection array for next-generation lithography (Figure 2) outlines the features of this soldering technique with respect to vacuum compatibility and very high component placement accuracy [7]. Silicon-based micro-structured MEMS devices for the deflection of multiple electron beams are precisely attached to ceramic carrier substrates utilizing both mechanical fixation and electrical contacting by the solder joint [8].
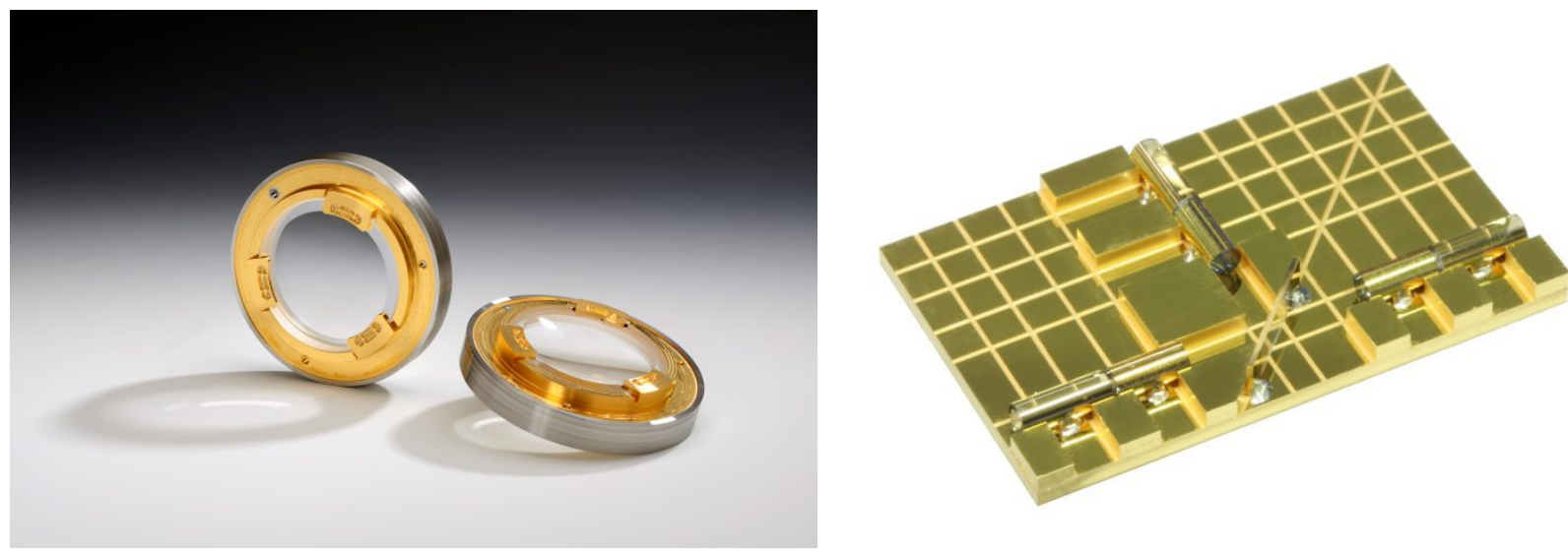

Figure 2: Examples of solderjet bumping assemblies: left, lens mount geometry soldered with cold soldering technique [9]; right, wavelength division multiplexing device for optical measurements [7].

The use of the Solderjet Bumping technique in the assembly of high-power lasers modules is reported in [10]. A variety of components from Fast-Axis-Collimator-lens (FAC) with very high accuracy demands to micro-structured beam splitting/combining units and beam shaping micro-lenses are mounted to a ceramics based Direct Copper Bond substrate (DCB).

\section{ASSEMBLY EXPERIMENTAL DETAILS}

For the diode-pumped solid-state laser for the ESA Exomars mission, the laser optical components are soldered on an aluminum nitride (AIN) ceramic substrate with copper for the heat-dissipative elements and Kovar pads used to reduce the thermal mismatch between the AIN substrate and the rest of the components. Two different redundant laser channels have to be assembled for the mission purposes. Each channel includes a laser diode $808 \mathrm{~nm}$, a couple of micro-lenses, an active medium laser crystal, second harmonic generator and a resonator mirror unit. The resonator mirror and the second harmonic generation crystal are mounted on a half sphere shape sub-mount to allow a highly precise tip/tilt alignment. The design also includes folding mirrors, a polarization coupling cube, a pinhole and a spliced fiber onto the output lens to guarantee a coaxial output beams (Figure 3). 


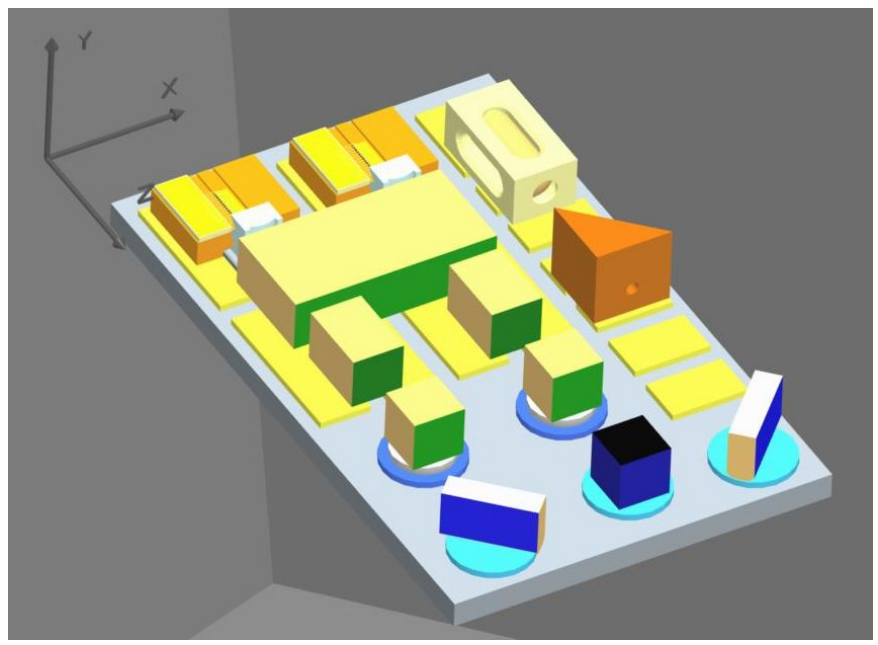

Figure 3: Exomars design of laser components soldered on an AIN ceramic of approximately $300 \mathrm{~mm}^{2}$.

Prior to the alignment and joining of the elements, all the components are metallized applying wettable surfaces by using DC magnetron sputtering Ti/Pt/Au. The first element to be soldered is the active medium laser crystal; after being metalized, an array of AuSn Solderjet Bumps (melting point $280{ }^{\circ} \mathrm{C}$ ) is applied on the surface to be soldered and later assembled by means of Fineplacer (multi-purpose bonder that offers a high placement accuracy). Although the solderjet technology proved to be better for soldering materials with different Coefficient of Thermal Expansion (CTE), the fine placer will assure a better operational laser cooling for this component. Subsequently, the diodes are placed in front of the crystal and soldered with SAC396 (melting point $217^{\circ} \mathrm{C}$ ) to the copper pads.

The rest of the optical components are soldered with different SAC305 solderjet bumps depending on the accuracy needed for each assembly [11]. The micro-lenses are aligned by a pneumatically-actuated gripper allowing 6 DOF, and soldered over the Kovar pad with the solderjet machine by two $300 \mu \mathrm{m}$ bumps placed on each side of the micro-lens. Afterwards, the second-harmonic generation crystal and the output coupler are aligned and soldered, both with a half sphere submount allowing accurate tip/tilt alignment and avoiding any translational movement in order to guarantee a soldering accuracy of a few arcseconds. The rest of the components (being less critical) are soldered over the pads using a $400 \mu \mathrm{m}$ solderjet bumps.

Finally, the laser is inserted inside a copper housing (Figure 4) specially designed and modeled ${ }^{1}$ for the mission purpose that will be hermetically sealed to ensure a leak smaller than $10^{-8} \mathrm{mbar} * 1 / \mathrm{s}$ by using the same cold soldering technique. Moreover, the housing is designed with an output Mini-AVIM ${ }^{2}$ connector (Figure 5) with a spherical lens that has to be soldered to the housing for the same hermeticity reasons. Also, the sphere has to be precisely assembled with SAC305 alloy to assure that the laser beam is coupled correctly to the output fiber.

1 Designs and Finite-Element-Method (FEM) analysis performed by LIDAX Ingeniería (Spain).

2 Manufactured for the mission purposes by Diamond SA (Switzerland). 

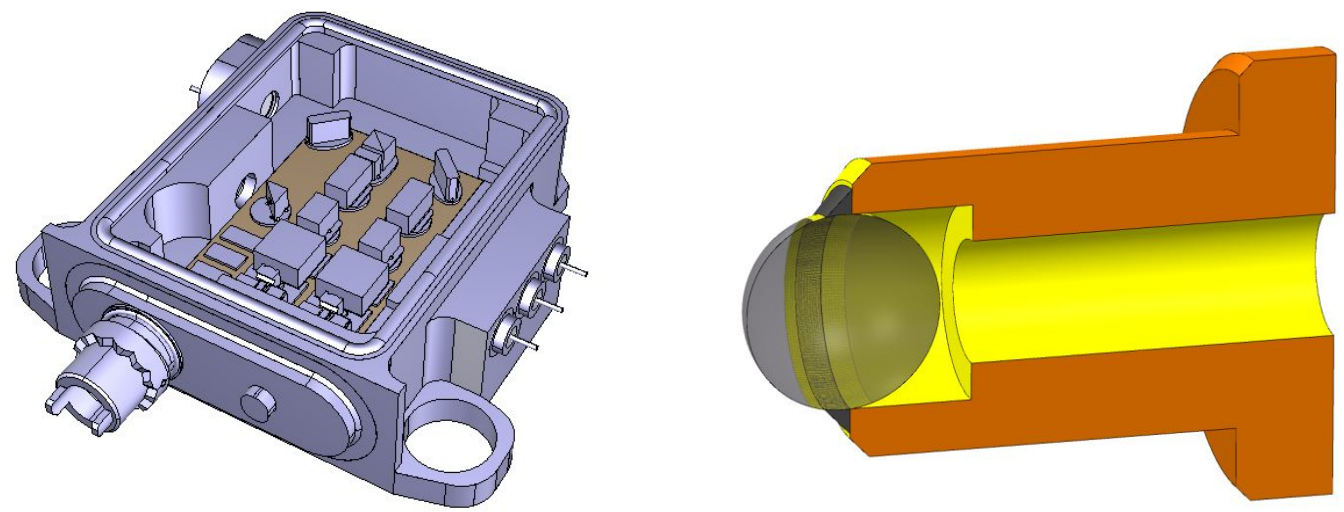

Figure 4: Left, design of the hermetically sealed housing by means of Solderjet technique (designs and simulations performed by LIDAX). Right, detail of the precisely assembled spherical fused silica lens into the stainless steel Mini-AVIM fiber connector, by means of cold soldering technique.

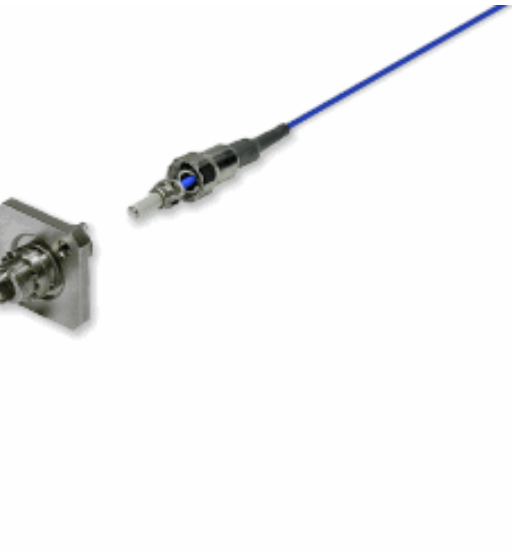

Figure 5: Example of Mini-AVIM connector specially designed for harsh environment as space applications. The Mini-AVIM combines two leading edge technologies: the AVIM MILstyle ratchet system and the base construction of Diamond Micro Interface (DMI) connector [12].

\section{RESULTS AND DISCUSSION}

Both adhesives and soldering techniques have been tested to assemble the laser unit for the Exomars ESA mission 2018. Different space-suitable adhesives have been studied (masterbond UV22, Masterbond EP21TDCHT-LOB, etc) to asses a better candidate, however none of them were free of gradual misalignments and instabilities over time. These misalignments have been observed during characterization time, making evaluation of a stable and repetitive laser emission impossible. Only the soldered assembled lasers by solderjet technology have been able to pass the stringent required specifications, being stable and constant over time.

The main tests performed on such lasers (at National Institute of Aerospace Technology INTA), able to be used in extreme conditions, are vibration, shock and thermal conditions. Through the mechanical characterization tests, the components have been proved to hold shear forces of 60 to $65 \mathrm{~N}$, this shear strength being enough to withstand a shock of more than $10^{6} \mathrm{~g}$ and able to handle the wide range of specified vibrational requirements for space devices. Even more, the assembled laser prototypes (Figure 6, left) were able to withstand the thermal environment requirements with a nonoperational temperature range from $-60{ }^{\circ} \mathrm{C}$ to $+70{ }^{\circ} \mathrm{C}$ and operational range between 15 to $45{ }^{\circ} \mathrm{C}$ (due to the strict wavelength stability requirement of a few picometers needed for Raman spectroscopy). Moreover, the devices were tested through radiation (proton and gamma radiation), vacuum and several burning and on-off cycles. 
The main requirements have been demonstrated before and after the different performed tests at constant temperature. The wavelength stability has been maintained, better than $\pm 0.005 \mathrm{~nm}$, the full width half maximum (FWHM) under $0.03 \mathrm{~nm}$ (Figure 6, right), a TEM00 spot has been stable in time and the required output power has been constant at $50 \mathrm{~mW}$, below a power consumption of $2 \mathrm{~W}$ (although the laser module can provide $>500 \mathrm{~mW}$ with a power consumption of around $4.5 \mathrm{~W}$ ).
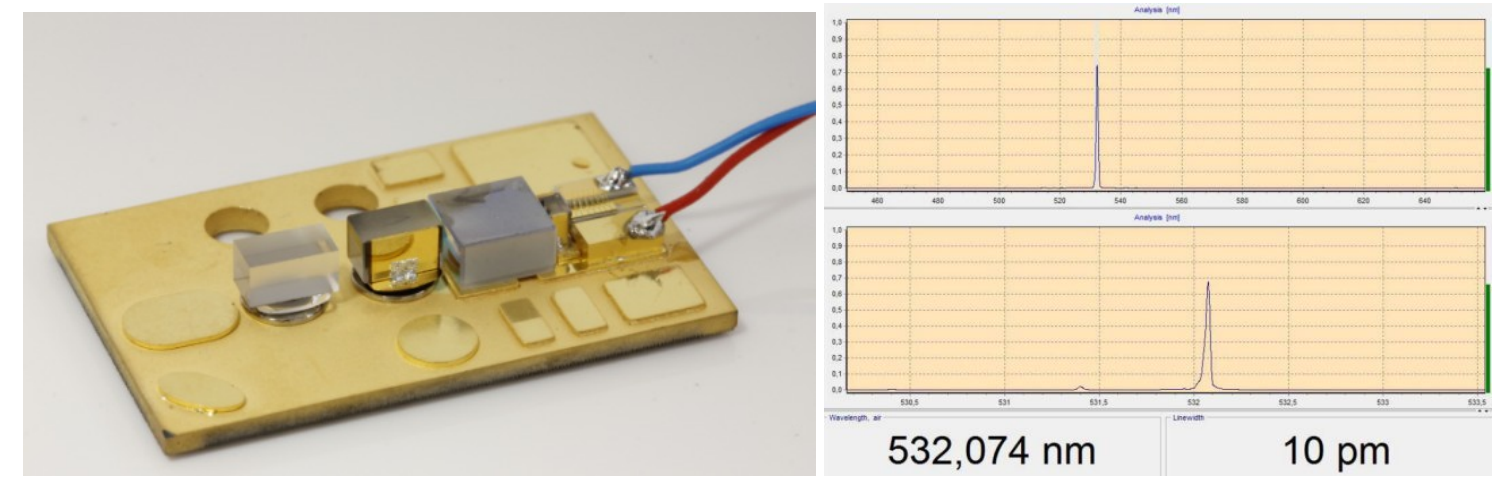

Figure 5: Left, soldered Exomars one laser channel resonator. Right, wavelength laser emission example.

\section{CONCLUSIONS}

A compact and miniaturized adhesive-free green laser for extreme conditions application has been achieved for the first time, applying laser-induced Solderjet bumping technology to fix the optical components in order to improve robustness and repeatability. This technology could be used in a wide range of applications that require similar characteristics as could be industry, medicine or military applications. Thanks to the 6 DOF SolderJet set-up, a wide range of geometries can be assembled to cover relevant design needs.

\section{ACKNOWLEDGEMENTS}

The soldering and assembling activities were performed within a framework agreement between Monocrom S.L. and Fraunhofer IOF. The work has been carried out within the ExoMars Raman instrument development activities of INTA. The characterization tests have been performed by Monocrom S.L and INTA. The Exomars Laser Spectrometer is a project funded by MINECO (Ministerio de Economía y Competitividad - Spain) and supervised and followed by INTA (Instituto Nacional de Tecnología Aeroespacial - Spain).

\section{REFERENCES}

[1] S. Ferrando, M. Galan, E. Mendez, E. Romeu, D. Montes, A. Isern, M. Jardi, J. Juliachs, G. Viera. "INNOVATIVE OPTICAL TECHNIQUES USED IN THE RAMAN INSTRUMENT FOR EXOMARS". ICSO International Conference on Space Optics, Greece (2010).

[2] F. Rull, S. Maurice, E. Diaz, C. Tato, A. Pacros and the RLS Team, "THE RAMAN LASER SPECTROMETER (RLS) ON THE EXOMARS 2018 ROVER MISSION". 42nd Lunar and Planetary Science Conference (2011).

[3] Banse, H., Beckert, E., Eberhardt, R., Stöckl, W. and Vogel, J., "Laser Beam Soldering - a New Assembly Technology for Microoptical Systems," Microsystems Technologies 11, pp. 186-193 (2005).

[4] Faidel, H., Gronloh, B., Winzen, M., Liermann, E., Esser, D., Morasch, V., Luttmann, J., Leers, M. and Hoffmann, D., "Passive alignment and soldering technique for optical components," Proc. SPIE 8235, 82351I (2012); doi:10.1117/12.906726. 
[5] Leers, M., Winzen, M., Liermann, E., Faidel, H., Westphalen, T., Miesner, J., Luttmann, J. and Hoffmann, D., "Highly precise and robust packaging of optical components," Proc. SPIE 8244, 824404 (2012); doi:10.1117/12. 920016.

[6] Beckert, E., Oppert, T., Azdasht, G., Zakel, E., Burkhardt, T., Hornaff, M., Kamm, A., Scheidig, I., Eberhardt, R. Tünnermann, A. and Buchmann, F., "Solder Jetting - A Versatile Packaging and Assembly Technology for Hybrid Photonics and Optoelectronical Systems," IMAPS $42^{\text {nd }}$ International Symposium on Microelectronics, Proceedings, 406-412 (2009).

[7] Burkhardt, T., Hornaff, M., Kamm, A., Rütz, M., Possner, T., Beckert, E., Eberhardt, R.and Tünnermann, A., "Assembly of a photonic wavelength-division multiplexing device using laser-based soldering," Proc. SPIE 8069, 80690V (2011); doi:10.1117/12.887001.

[8] Burkhardt, T., Mohaupt, M., Hornaff, M., Zaage, B., Beckert, E., Döring, H.-J., Slodowski, M., Reimer, K., Witt, M., Eberhardt, R. and Tünnermann, A., "Packaging Technology of Multi Deflection Arrays for Multi-Shaped Beam Lithography," IMAPS $44^{\text {th }}$ International Symposium on Microelectronics, Proceedings, 600-607 (2011).

[9] Burkhardt, T., Hornaff, M., Ribes, P., Kamm, A, Burkhardt, D., Kousar, S., Beckert, E., Eberhardt, R. and Tünnermann, A., "Laserlöten optischer Systeme - Anwendungen des Solderjet Bumpings," 9. Jenaer Lasertagung 2014, in: DVS-Berichte Band 370, Innovative Fertigung durch Lasersysteme neuester Generation, Vorträge und Posterbeiträge der 9. Jenaer Lasertagung am 20. und 21. November 2014, DVS Media, Düsseldorf, 2014, ISBN 9783-945023-04-4, ISSN 0418-9639.

[10] Beckert, E., Schreiber, P., Burkhardt, T., Werner, E. and Hülsewede, R., "Design and assembly of a miniaturized high-power laser bar to 50 um fiber coupling module," Proc. SPIE 6876, 687613 (2008).

[11] Burkhardt, T., Beckert, E., Hornaff, M., Thiele, J., Burkhardt, D., Galan, M., Gilaberte, M., Ferrando, S., Montes, D., Ribes, P., Eberhardt, R. and Tünnermann, A., "Solder joining of a miniaturized laser on a multi-material smart platform," Proceedings of the $6^{\text {th }}$ Smart Systems Integration, (2012).

[12] Diamond SA, "Mini-AVIM-Family", http://www.diamond-fo.com/en/products/Assemblies---Adapters/standardsingle-fiber-based/Mini-AVIM-Family 